\title{
A Double Jaw Hand Designed for Multi-object Assembly
}

\author{
Joshua C. Triyonoputro ${ }^{1}$, Weiwei Wan ${ }^{1,2, *}$, Kensuke Harada ${ }^{1,2}$
}

\begin{abstract}
This paper presents a double jaw hand for industrial assembly. The hand comprises two orthogonal parallel grippers with different mechanisms. The inner gripper is made of a crank-slider mechanism which is compact and able to firmly hold objects like shafts. The outer gripper is made of a parallelogram that has large stroke to hold big objects like pulleys. The two grippers are connected by a prismatic joint along the hand's approaching vector. The hand is able to hold two objects and perform in-hand manipulation like pull-in (insertion) and push-out (ejection). This paper presents the detailed design and implementation of the hand, and demonstrates the advantages by performing experiments on two sets of peg-in-multi-hole assembly tasks as parts of the World Robot Challenge (WRC) 2018 using a bimanual robot.
\end{abstract}

Index Terms-Assembly, grippers, grasping, in-hand manipulation.

\section{INTRODUCTION}

One major challenge of next-generation manufacturing is autonomous assembly. Many studies have been devoted to related problems in the past decades [1], [2], [3]. Most of them used parallel grippers to plan grasps and assembly sequences. Although they made impressive progress, an inherent problem remains - holding and manipulating multiple objects simultaneously. Fig,1 (a) shows an example where a human hand holds and manipulates two objects (a peg and a pulley) together. The task is widely seen in realworld product assembly and difficult to be performed using simple parallel grippers.

There are several possible hand design solutions to the remaining problem. For example, some designs used two or more grippers in one robotic hand [4]. The grippers can be fixed to tuning turrets [5], or they can have one or more Degree of Freedoms (DoFs) relative to each other [6], [7]. Some other designs used fully actuated [8] or underactutated anthropomorphic hands [9], [10], [11]. Specifically, Zeng et al. [6] developed a gripper with a retractable mechanism to allow switching between a parallel gripper and a suction gripper. Cannella et al. [12] and Chen et al. [13] developed industrial grippers with twisting ability for high-speed assembly. Ma et al. [14] developed a two-finger gripper using an underactuated human-like finger and a passive thumb. It is capable of performing in-hand manipulation like pull-in and push-out. Odhner et al. [15] developed a 3-finger underactuated hand using a minimalistic design optimized for a set of tasks. Yamaguchi et al. [16] added dexterity to underactuated fingers by adding suction cups on each fingertip. Kakogawa et al. [17] developed an under-actuated three-finger gripper with pull-in ability. Chavan-Dafle et al. [18] used a 3finger one-parameter gripper to extrinsically manipulate

${ }^{1}$ Graduate School of Engineering Science, Osaka University, Japan. ${ }^{2}$ National Inst. of AIST, Japan. *Correspondent author: Weiwei Wan wan@hlab.sys.es.osaka-u.ac.jp

${ }^{a}$ http://worldrobotsummit.org/en/programs/challenge/
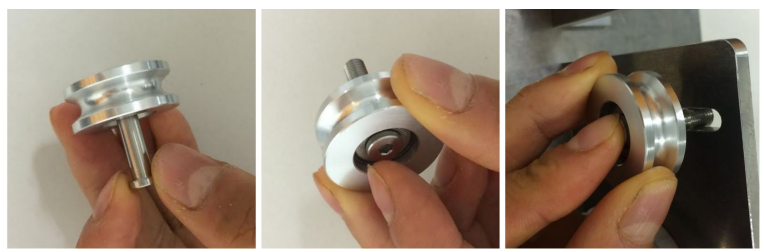

(a) A human hand holding and manipulating two objects with one hand in product assembly.
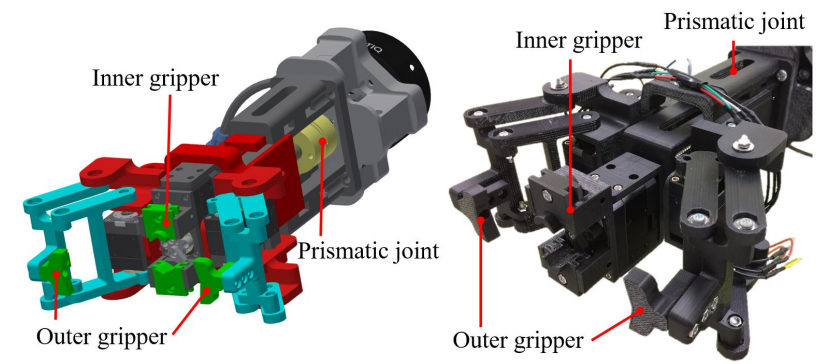

(b) CAD models of the double jaw hand and an implementation.

Fig. 1: Motivations of the work and the developed hand.

objects. The finger shape of the gripper was optimized for holding spherical objects [19]. These designs suggested general solutions to tackle the problem of holding and manipulating multiple objects simultaneously, but they do not fully address the undergoing details. They either only solve the problem partially or have special mechanisms that decrease the robustness of robotic systems.

The inherent problem and the drawbacks of the available hand designs inspire us to develop a more dexterous but still simple robotic hand for autonomous assembly. The requirements of the new hand are as follows.

1) Simple mechanisms and small number of actuators

2) Capable of holding two objects

3) Capable of in-hand pull-in and push-out

4) Capable of aligning objects along a common axis

5) Large stroke and large holding force

In response to the requirements, we propose a double jaw hand that satisfies these requirements. The CAD models and an implementation are shown in Fig 1 (b). The hand is made of two orthogonal parallel grippers with different mechanisms. The inner gripper is made of a crankslider mechanism which is compact and able to firmly hold objects like shafts. The outer gripper is made of a parallelogram that has large stroke to hold big objects like pulleys. The two grippers are connected by a prismatic joint along the hand's approaching vector. The hand is able to hold two objects and perform in-hand manipulation like pull-in (insertion) and push-out (ejection).

The design is verified with real-world executions of peg-in-multi-hole assembly tasks using objects from the World Robotic Challenge (WRC) 2018 assembly challenge. Experiments are also performed to show that the hand is 
able to align a peg and a pulley, and assemble the peg into the pulley without external help.

The paper is organized as follows. Section $\Pi$ explains in details the motivations and the requirements for designing the hand. Section III presents the details of the gripping mechanisms and implementations. Section IV]demonstrates the efficacy of the developed hand using two tasks from the WRC2018 assembly challenge. Section V] draws conclusions and presents future work.

\section{Motivations}

The proposed design is mainly motivated by the tasks in WRC2018 assembly challenge.

WRC2018 assembly challenge requires using robots to autonomously assemble a Belt Drive Unit shown in Fig 2(b). This paper focuses on tackling one aspect of the whole task - peg-in-multi-hole assembly. Peg-in-multihole assembly is an extension to peg-in-hole assembly. The goal of peg-in-hole assembly is to insert a peg into a hole [20], [21], [22], while the goal of a peg-in-multi-hole assembly is to continuously insert peg-and-hole complex into other holes. In the WRC2018 assembly challenge, robots have to perform several sets of peg-in-multi-hole assembly subtasks. Two of them, namely the clamping pulley set and the idle pulley set, are shown in Fig 2 a). The clamping pulley set comprises inserting a pulley shaft into a clamping pulley, inserting the shaft-and-pulley complex into the hole of a pulley shaft spacer, and inserting the shaft-pulley-spacer complex into the hole of a housing. The idle pulley set comprises inserting a retainer pin into an idle pulley, inserting the pin-and-pulley complex into a retainer pin spacer, and inserting the pin-pulley-spacer complex into a slot.

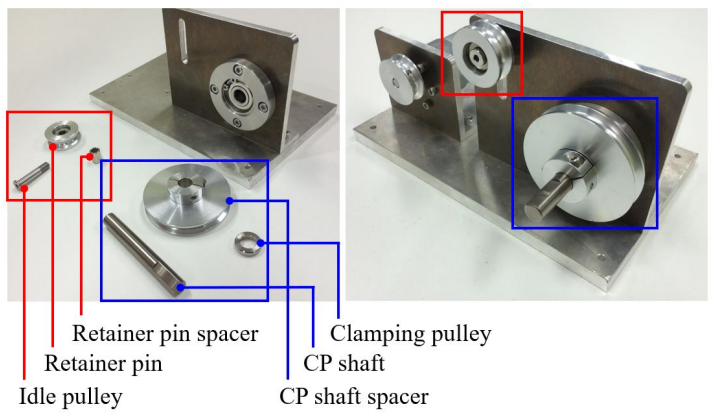

Fig. 2: Objects used in the peg-in-multi-hole assembly tasks of the WRC 2018 assembly challenge. The objects in red and blue boxes will be assembled to a base respectively.

Essentially, the two sets of peg-in-multi-hole assembly share the same workflow, which involves (a) inserting a peg to the hole of a pulley, (b) inserting the peg-andpulley complex to the hole of a spacer, (c) inserting the peg-pulley-spacer complex into the hole on a metal base. Illustrations of human assembling the two sets using the workflow are shown in Fig. 3 and Fig. 4 respectively.

In order for a robotic hand to perform similar tasks, it must meet the following requirements. First, the hand must be able to hold two objects to avoid slipping while performing assembly. In Fig. 3 b, c), the inserted shaft must

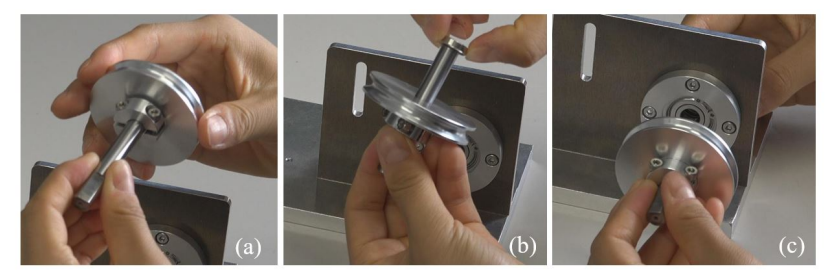

Fig. 3: Manually assembling the clamping pulley set. (a) Peg-in-hole: Insert a shaft into the hole of a pulley. (2) Complex-in-hole: Inserting the shaft-and-pulley complex into the hole of a spacer. (3) Complex-in-hold: Insert the shaft-pulley-spacer complex into the hole of a bearing.
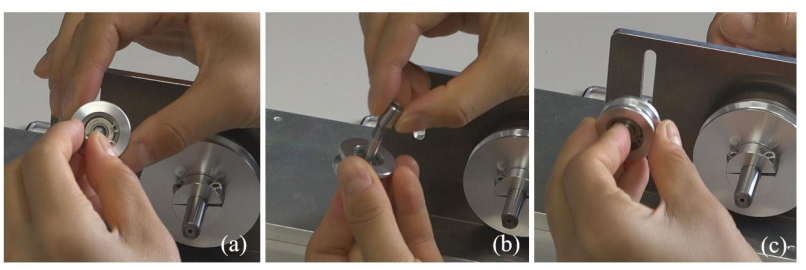

Fig. 4: Manually assembling the idle pulley set. (a) Pegin-hole. (b) Complex-in-hole: Insert the pin-and-pulley complex into the hole of a spacer. (3) Complex-in-hole: Insert the pin-pulley-spacer complex into a slot on the base.

be held together with the pulley and spacer after being inserted. Or else, the pulley and spacer may slip out of the complex when the robot rotates its arm to move from one key pose to another, leading failure in assembly. Another example is inserting the shaft into the pulley. It is possible to finish the task using two arms with simple grippers: One arm holds the clamping pulley, and the other holds the pulley shaft. The pulley shaft is inserted into the pulley using impedance control. However, the two arms in this configuration cannot directly continue to the next peg-inhole step after finishing the insertion. It has to perform some regrasps and handovers: First, one arm releases the pulley shaft while the clamping pulley is held by the other arm. Then, the shaft-and-pulley complex is handed over to the free arm. Third, the two arms perform another handover to let the previous arm hold one end of the shaft. The process is time consuming and vulnerable. Errors and noises accumulate during the multiple times of regrasp and handover, leading to failure in assembly. Developing a hand that can hold two objects could avoid these problems. For one thing, it could hold both objects to avoid slipping. For the other, it could minimize the necessary deliberate motion design or motion planning by switching grippers using in-hand commands.

Second, the hand must be able to align objects along a common axis. Before inserting a peg into a hole, a process is needed to calibrate the peg and the hole using vision systems or spiral/linear search. The calibration and searching process is time consuming. A better solution could be holding the two objects, align them in-hand to correct the positions of a peg and a hole. Aligning objects along a common axis is not used in the human demonstration in Fig 3 and Fig 4

Third, a robotic hand that can perform similar tasks must 
be able to perform in-hand manipulation like pull-in and push out. Fig. 4(b) shows that because the retainer pin is short, it is pushed against the idle pulley by one finger while another two fingers grasp the pulley to prepare for inserting into the spacer. The process cannot be done by a single gripper or grippers installed on multiple robotic arms like [2], [3]. Instead, the gripper needs to be able to perform in-hand manipulation. It needs to grasp both the retainer pin and the idle pulley, push the retainer pin into the pulley using in-hand manipulation, and switch to multiobject holding mode before inserting them into the spacer and slot.

These analysis led us to explore the possibility of performing insertion of a peg into a pulley using a double jaw design. Our expectation is two robotic hands, one is the double jaw hand, the other is a simple gripper, are installed to a dual-arm robot to perform peg-in-multi-hole tasks. The double jaw hand could hold two objects, align them, and assemble those two objects into a complex using in-hand pull-in and push-out. It could also cooperate with the simple gripper on another arm to perform similar pegin-hole tasks and insert the complex into other holes held.

\section{GRIPPER DESIGN}

In this section, an explanation of the design of the double jaw hand is given. The double jaw hand is consisted of two parallel grippers that are controlled independently, share the same approaching vector, and are connected by a prismatic joint.

The hand has four DoFs driven by four motors. Robotis XM430-350-R servo motors were used because they have a high maximum torque of $4.1 \mathrm{Nm}(12 \mathrm{~V})$, a relatively small size, and a built-in control system that allows users switch between position, velocity, and force control. Details of the inner gripper, the outer gripper, and the prismatic joint are as follows.

\section{A. Inner gripper}

The inner gripper is a parallel gripper controlled using one Robotis XM430-350-R motor. The opening and closing motion is shown on Fig. 5(a). The gripper is actuated by a crank-slider mechanism which is simple and transmits a large force for holding objects firmly.

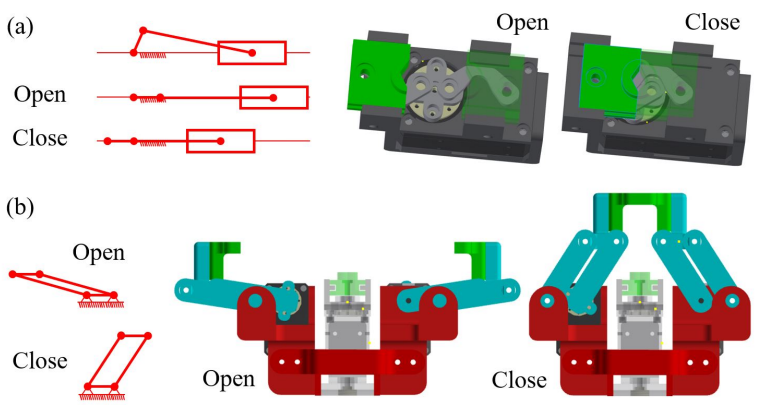

Fig. 5: (a) Mechanism of the inner gripper. It is essentially a slide crank shown in the left. (b) Mechanism of the outer gripper. It is essentially a parallelogram.

The maximum stroke of the inner gripper is about 20 $\mathrm{mm}$. The maximum stroke is small because it is made to precisely and firmly grasp objects like the pulley shaft and the retainer pin shown in Fig 2. Here, the pulley shaft has a diameter of $10 \mathrm{~mm}$. The retainer pin has a diameter of $6 \mathrm{~mm}$ and larger head with a diameter of $9 \mathrm{~mm}$.

The fingertips of the inner gripper are cut into vshapes since the target objects are generally cylindrical in shape. The v-shape fingertips also help to align objects. In addition, to account for the larger head of the retainer pin, the bottom part of the fingertips has a slightly larger v-shape cut.

\section{B. Outer gripper}

The outer gripper is also a parallel gripper. The mechanism used for transmission is a parallelogram. The gripper has two DoFs. Each finger is controlled using one Robotis XM430-350-R motor, and the two fingers can move independently. Fig. 5 (b) shows the open and close motion of the outer gripper. Although not shown in the figure, the two fingers can also move independently. The stroke of the outer gripper is $150 \mathrm{~mm}$, which is able to hold the large pulleys shown in Fig 2. The fingertips of the outer gripper are also cut into v-shapes to align cylindrical objects.

There are two main reasons for making the outer gripper has two DoF. First, it is to keep the middle part empty. The middle part must be left empty to prepare space for the prismatic joint to connect inner and outer gripper. Second, it is to keep the hand symmetric in mass distribution. Symmetric design will facilitate impedance control in dual-arm assembly. Third, 2DoFs allow non-synchronous motion, which might be useful for later study. It is therefore advisable to install two independent motors sideways.

\section{Prismatic Joint}

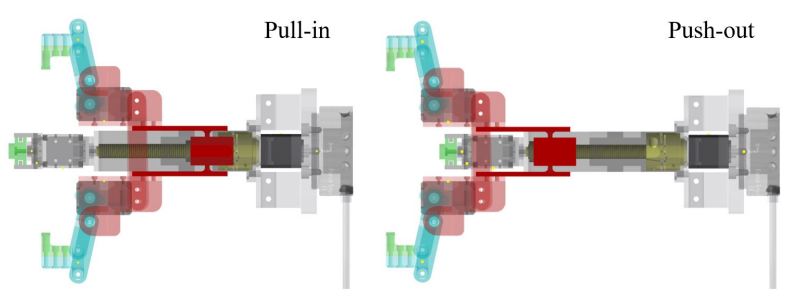

Fig. 6: Motion of the prismatic joint. The prismatic joint is essentially a linear screw.

The prismatic joint is used to connect the two parallel grippers together along the same approaching vector. We added a prismatic joint because this allows for the two grippers to have one DoF relative to each other. The addition of a prismatic joint enables in-hand pull-in and push-out, making one hand peg-in-hole possible.

The mechanism of the prismatic joint is a linear screw. The nut of the linear screw is connected to the outer gripper. The lead is coupled to one motor. Fig 6 shows the motion of the prismatic joint. The Robotis XM430350-R motor allows close-loop control, which can stop the linear screw in the presence of mechanical stoppers. The prismatic joint is calibrated such that $0 \mathrm{~mm}$ refers to the position when the outer gripper is completely pulled-in (left, Fig 6). $73 \mathrm{~mm}$ refers to the maximum position when the prismatic joint is completely pushed-out (right, Fig6). 


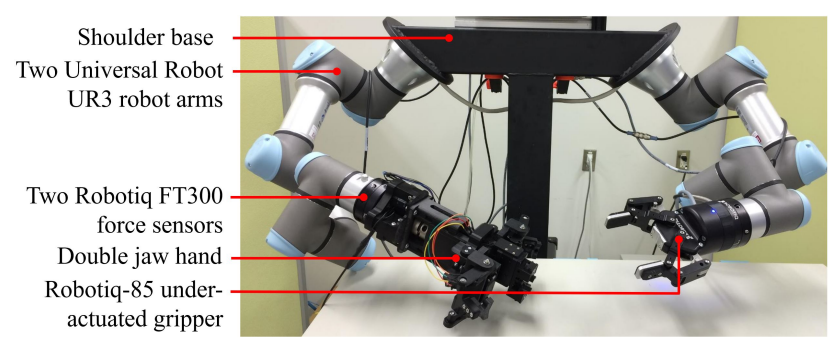

Fig. 7: Bimanual robot platform used to conduct the experiments. Force sensors are used for impedance control.

\section{EXPERIMENTS AND DiscusSION}

Two sets of peg-in-multi-hole tasks in the WRC2018 assembly challenge are performed using a bimanual robot. Fig.7 shows the configurations of the bimanual robot. It comprises two Universal Robot UR3 robotic arms attached to a shoulder base. Two Robotiq FT300 force sensors were attached to the end of the two arms. The double jaw hand developed in this paper is attached to the force sensor on to the right arm. The Robotiq-85 two-finger adaptive gripper is attached to the force sensor on the left arm.

In this section we present the details of peg-in-multihole experiments performed by the dual arm robot using our dual jaw hand. We also discuss about the experiments of using the double jaw hand to insert a peg into a pulley with in-hand manipulation.

\section{A. Experiments}

1) Peg-in-multi-hole assembly of the clamping pulley set and the idle pulley set: This subsection shows an explanation on the results of the two sets of peg-in-multi-hole assembly. The experimental flow is as follows. First, we manually made the double jaw hand and the parallel gripper grasp the objects for the targeted peg-in-hole assembly. After that, we ran a routine which consists of linear search, spiral search, and insertion using impedance control to push the pegs into holes. Linear search means making the gripper holding a peg moves towards the other gripper until the peg hits an obstacle. The robot stops the linear motion in the presence of obstacles. Spiral search refers to the motion of repeatedly trying to locate a hole while changing the position of the end of the peg. The robot draws circles of increasingly larger radius until it finds a hole. This is necessary to account for imprecision. Once the robot finds the location of the hole, it inserts the peg into the hole using impedance control. The insertion process stops until the robot are moved to a given pose. After successful insertion, we manually controlled the motions of the two grippers and prepare them for the following complex-in-hole assembly.

It is true that the entire motion sequences, including picking objects from a given spot accurately with the help of visual sensors, is an important part of the entire peg-inmulti-hole assembly. Nevertheless, given that the focus of these experiments were to test the capability of the double jaw hand to grasp multiple objects and manipulate these objects along a common axis using a prismatic joint, the picking parts of the experiments are to be performed as part of the future work. Similarly, while automation of motions of the robot and the grippers is important part of the research, its automation does not directly relate to the focus of the paper, therefore we decided to do it in the future. One aspect that was not manually adjusted was the position of the robot arms after it moved to the starting position prior to a peg-in-hole motion. This was to make the spiral search observable.

Fig 8 shows images sequences of the robot performing the peg-in-multi-hole assembly of the clamping pulley set. In Fig 8 (a), the parallel gripper installed to the left arm holds the pulley shaft and inserts it to a clamping pulley held by the double jaw hand to minimize the need for two handovers. In the Fig $8(\mathrm{~b}, \mathrm{c})$, the shaft-pulley complex is held by the double jaw hand. The double jaw hand inserts the complex into the hole of a pulley shaft spacer and the hole of a bearing.

Fig 9 shows image sequences of the robot performing peg-in-multi-hole assembly of the idle pulley set. Compared to inserting the pulley shaft into the clamping pulley, inserting the retainer pin to the idle pulley was more complicated since the pin must be fully embedded in the pulley. The process is divided into two steps, including an insertion step and an in-hand manipulation step (from grasping the retainer pin to pushing the retainer pin). Fig 9 (a) shows the two steps. The two complex-in-hole assembly in Fig 9 (b, c) are similar as the ones from the clamping pulley set.

The results of the experiments after performing multiple trials of the peg-in-multi-hole assembly are as follows. The first step of the three-step peg-in-multi-hole assembly is generally successful, even though it takes some time for the robot to locate a hole. The second step of the peg-inmulti-hole assembly is the one which may fail depending on how the spacer becomes increasingly tilted every time the peg hits it during spiral search. The third step is also generally successful.

2) Insertion of a peg to a pulley using the double jaw gripper: In this subsection, we examine the possibility of using double jaw hand to align a peg and a pulley and perform insertion by pulling-in prismatic joint. Fig 10 shows the result of the experiments performed on the retainer pin and the idle pulley. We managed to conduct this experiment successfully repeatedly with the retainer pin and the idle pulley, but the experiments are largely not successful for the pulley shaft and the clamping pulley. This is probably because the clamp part and the pulley part of the clamping pulley have different hole sizes. The insertion is performed such that the peg entered first to the protruding clamp part, otherwise it would end up requiring handovers. While insertion into the clamp part is largely successful, the peg easily hits the pulley part and gets stuck, leading to failure in assembly. This result is like our expectation because it shares the similar reason as to why the insertion such as in Fig 8 a) is chosen instead of insertion to the other side (clamp part) of the clamping pulley.

\section{B. Discussion}

First, we discuss how the experiments verified the requirements on gripper design stated in Section $\mathrm{I}$ and Section II Throughout the experiments, the hand is performing 


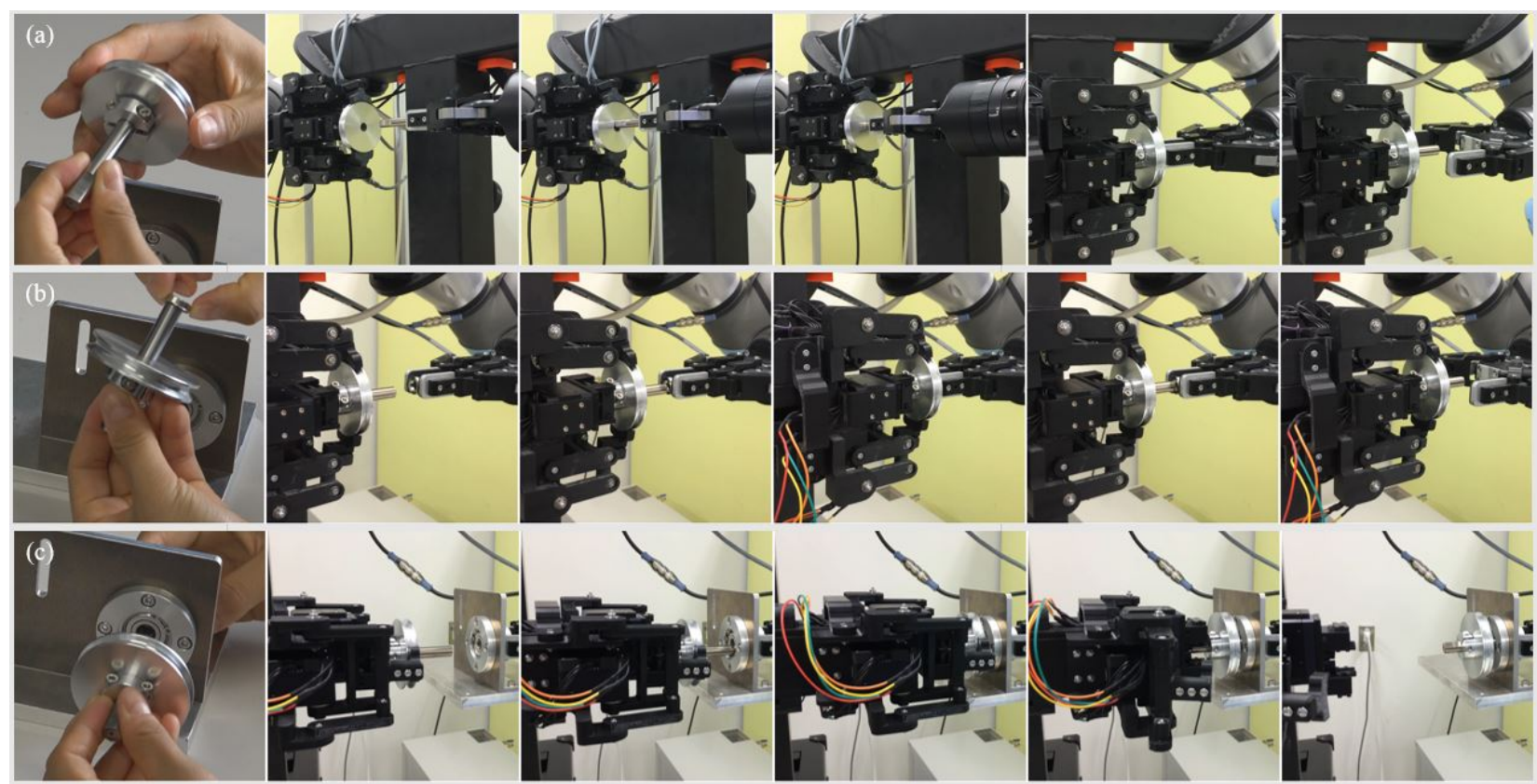

Fig. 8: Peg-in-multi-hole assembly experiments on the clamping pulley set. (a) Insertion of the pulley shaft into the clamping pulley. (b) Insertion of a complex (the pulley shaft and the clamping pulley) into the pulley shaft spacer. (c) Insertion of a complex (the pulley shaft, the clamping pulley, and the pulley shaft spacer) into the bearing on the base.

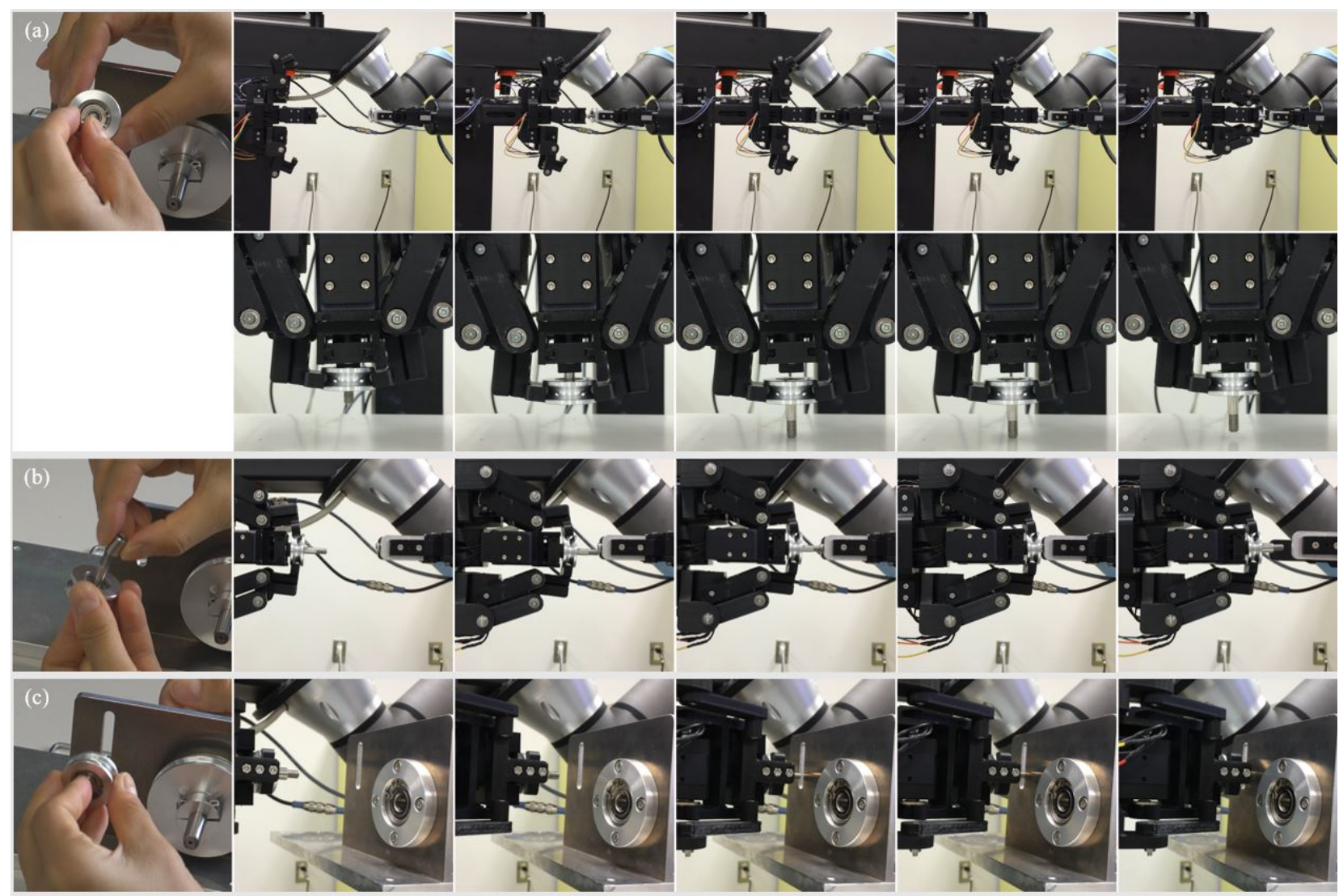

Fig. 9: Peg-in-multi-hole assembly experiments on the idle pulley set. (a) Upper row: Insertion of the retainer pin into the idle pulley; Lower row: In-hand manipulation to prepare for the next peg-in-hole assembly. (b) Insertion of a complex (the retainer pin and the idle pulley) into the retainer pin spacer. (c) Insertion of a complex (the retainer pin, the idle pulley, the retainer pin spacer) into a slot on the base. 


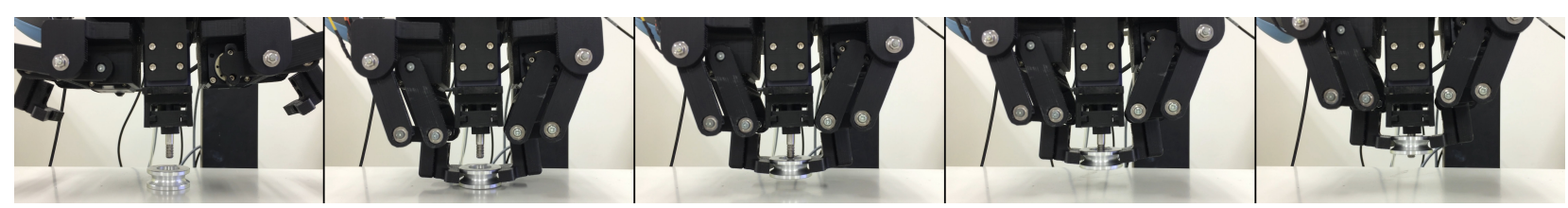

Fig. 10: Insertion of the retainer pin to the idle pulley using in-hand manipulation.

tasks like complex-in-hole, demonstrating that the hand could hold two objects. At the end of the first peg-in-hole between the retainer pin and the idle pulley, the double jaw hand held both objects. The gripper continued to release the retainer pin and push the retainer pin to keep it in place while holding the idle pulley. This shows the intrinsic inhand manipulation capability of the gripper. The clearance between the retainer pin and the idler pulley is $0.01 \mathrm{~mm}$, indicating that the hand is good at aligning objects. The outer gripper also has enough stroke to hold large objects.

Second, we discuss the shortcomings of the developed gripper. The two grippers of the double jaw hand are connected by a linear screw. Given that predefined settings in Robotis XM430-350-R do not allow users to control the rotational speed in the current-based position control mode which is used to control the prismatic joint, the prismatic joint movement is slow. It takes about 55 seconds to move from $0 \mathrm{~mm}$ to $73 \mathrm{~mm}$, which means that the linear speed is approximately $1.3 \mathrm{~mm} /$ second. This restricts the efficiency of peg-in-hole assembly using in-hand pull-in and pushout.

\section{Conclusions and Future Work}

This paper presented a novel double jaw hand capable of holding two objects and performing in-hand manipulation. Experimental and real-world execution results showed that the hand is simple, robust, and is able to perform peg-inmulti-hole assembly with the help of an external gripper. Our future work includes optimizing the size of the double jaw hand and developing automatic grasp planning and control algorithms.

\section{ACKNOWLEDGEMENT}

This paper is based on results obtained from a project commisioned by the New Energy and Industrial Technology Development Organisation (NEDO). The authors would like to thank Felix von Drigalski and Chisato Nakashima from OMRON SINIC X and fellow members of Robotics Manipulation Laboratory, Osaka University for their support in the development of this work.

\section{REFERENCES}

[1] A. Hormann and U. Rembold, "Development of an advanced robot for autonomous assembly," in Robotics and Automation, 1991. Proceedings., 1991 IEEE International Conference on. IEEE, 1991, pp. 2452-2457.

[2] M. Dogar, A. Spielberg, S. Baker, and D. Rus, "Multi-robot grasp planning for sequential assembly operations," in Robotics and $\mathrm{Au}$ tomation (ICRA), 2015 IEEE International Conference on. IEEE, 2015, pp. 193-200.

[3] W. Wan, F. Lu, Z. Wu, and K. Harada, "Teaching robots to do object assembly using multi-modal $3 \mathrm{~d}$ vision," Neurocomputing, vol. 259, pp. 85-93, 2017.

[4] G. J. Monkman, S. Hesse, R. Steinmann, and H. Schunk, Robot grippers. John Wiley \& Sons, 2007.
[5] M. T. Mason, Mechanics of Robotic Manipulation. The MIT Press, 2001.

[6] A. Zeng, S. Song, K.-T. Yu, E. Donlon, F. R. Hogan, M. Bauza, D. Ma, O. Taylor, M. Liu, E. Romo et al., "Robotic pick-and-place of novel objects in clutter with multi-affordance grasping and crossdomain image matching," arXiv preprint arXiv:1710.01330, 2017.

[7] T. Atakuru and E. Samur, "A robotic gripper for picking up two objects simultaneously," Mechanism and Machine Theory, vol. 121, pp. 583-597, 2018.

[8] Z. Xu and E. Todorov, "Design of a highly biomimetic anthropomorphic robotic hand towards artificial limb regeneration," in Robotics and Automation (ICRA), 2016 IEEE International Conference on. IEEE, 2016, pp. 3485-3492.

[9] W. Townsend, "The barretthand grasper-programmably flexible part handling and assembly," Industrial Robot: an international journal, vol. 27, no. 3, pp. 181-188, 2000.

[10] B. Massa, S. Roccella, M. C. Carrozza, and P. Dario, "Design and development of an underactuated prosthetic hand," in Robotics and Automation, 2002. Proceedings. ICRA'02. IEEE International Conference on, vol. 4. IEEE, 2002, pp. 3374-3379.

[11] R. Deimel and O. Brock, "A novel type of compliant and underactuated robotic hand for dexterous grasping," The International Journal of Robotics Research, vol. 35, no. 1-3, pp. 161-185, 2016.

[12] F. Cannella, F. Chen, C. Canali, A. Eytan, A. Bottero, and D. Caldwell, "Design of an industrial robotic gripper for precise twisting and positioning in high-speed assembly," in System Integration (SII), 2013 IEEE/SICE International Symposium on. IEEE, 2013, pp. 443-448.

[13] F. Chen, F. Cannella, C. Canali, T. Hauptman, G. Sofia, and D. Caldwell, "In-hand precise twisting and positioning by a novel dexterous robotic gripper for industrial high-speed assembly," in Robotics and Automation (ICRA), 2014 IEEE International Conference on. IEEE, 2014, pp. 270-275.

[14] R. R. Ma, A. Spiers, and A. M. Dollar, "M 2 gripper: Extending the dexterity of a simple, underactuated gripper," in Advances in reconfigurable mechanisms and robots II. Springer, 2016, pp. 795805.

[15] L. U. Odhner, L. P. Jentoft, M. R. Claffee, N. Corson, Y. Tenzer, R. R. Ma, M. Buehler, R. Kohout, R. D. Howe, and A. M. Dollar, "A compliant, underactuated hand for robust manipulation," The International Journal of Robotics Research, vol. 33, no. 5, pp. 736752,2014

[16] K. Yamaguchi, Y. Hirata, and K. Kosuge, "Development of robot hand with suction mechanism for robust and dexterous grasping," in Intelligent Robots and Systems (IROS), 2013 IEEE/RSJ International Conference on. IEEE, 2013, pp. 5500-5505.

[17] A. Kakogawa, H. Nishimura, and S. Ma, "Underactuated modular finger with pull-in mechanism for a robotic gripper," in Robotics and Biomimetics (ROBIO), 2016 IEEE International Conference on. IEEE, 2016, pp. 556-561.

[18] N. Chavan-Dafle, A. Rodriguez, R. Paolini, B. Tang, S. S. Srinivasa, M. Erdmann, M. T. Mason, I. Lundberg, H. Staab, and T. Fuhlbrigge, "Extrinsic dexterity: In-hand manipulation with external forces," in Robotics and Automation (ICRA), 2014 IEEE International Conference on. IEEE, 2014, pp. 1578-1585.

[19] A. Rodriguez and M. T. Mason, "Effector form design for 1dof planar actuation," in Robotics and Automation (ICRA), 2013 IEEE International Conference on. IEEE, 2013, pp. 349-356.

[20] H. Inoue, "Computer controlled bilateral manipulator," Bulletin of $J S M E$, vol. 14, no. 69, pp. 199-207, 1971.

[21] M. T. Mason, "Compliance and force control for computer controlled manipulators," IEEE Transactions on Systems, Man, and Cybernetics, vol. 11, no. 6, pp. 418-432, 1981.

[22] Y. Zheng, X. Zhang, Y. Chen, and Y. Huang, "Peg-in-hole assembly based on hybrid vision/force guidance and dual-arm coordination,' in Robotics and Biomimetics (ROBIO), 2017 IEEE International Conference on. IEEE, 2017, pp. 418-423. 